\title{
Systems level modelling of metabolism in fungal endophytes - implications for the symbiosis with ryegrass
}

\author{
RAJIV CHATURVEDI ${ }^{1}$, TANYA SOBOLEVA ${ }^{1}$, LINDA J. JOHNSON ${ }^{2}$, \\ ANTHONY J. PARSONS ${ }^{2}$ and SUSANNE RASMUSSEN ${ }^{2}$ \\ ${ }^{1}$ AgResearch Limited, Ruakura Research Centre, Private Bag 3123, East Street, Hamilton 3240, New Zealand \\ ${ }^{2}$ AgResearch Limited, Grasslands Research Centre, Tennent Drive, Private Bag 11008, Palmerston North 4442, New Zealand
} rajiv.chaturvedi@agresearch.co.nz

\begin{abstract}
We used constraint based stoichiometric modelling of metabolic fluxes in Epichloë festucae (FL1), a targeted gene replacement of a non-ribosomal peptide synthetase (termed sidF) from $E$. festucae, and the symbiotic association of these endophytic fungi and their host Lolium perenne. SidF encodes an excreted ironchelating siderophore and the $s i d F$ knockouts $(\mathrm{KO})$ are impaired in their ability to take up iron. After constructing the metabolic network at a genome scale, we applied constraints on enzymatic reactions that require iron as co-factor to study the variations in metabolic network capabilities of the siderophore mutant versus wildtype, in culture and in planta. We compared fluxes calculated for the production of amino acids with observed concentrations of these amino acids in planta. We report a counter-intuitive result from considering metabolism on a systems level in our models.

Keywords: stoichiometric metabolic network modelling, flux balance analysis, symbiosis, Neotyphodium lolii, Lolium perenne, Epichloë festucae.
\end{abstract}

\section{Introduction}

An aim of systems biology is to integrate all levels of information to understand how biological systems function as a whole; the discipline hence seeks theoretical means to deal with the "omes" (Mustacchi et al. 2006). Wide use of high throughput technologies has made large amounts of data on the genome, transcriptome and metabolome scale available. The question arises: Does the mass of data itself allow us to understand characteristics of an organism and how its function is regulated by the combined influences of the genome, environment, and evolutionary selection? If there is one thing these data tell us, it is the necessity to consider the metabolism of the cell in its entirety, since the complex interconnections in the metabolic network of a cell preclude a one-to-one correspondence between the genotype and the phenotype. In order to quantitatively describe cell physiology as a function of the activity of multiple gene products and environmental factors, with proper account taken of the interrelatedness and connectivity of these objects, we need the language of mathematical modelling to express the biochemical reactions that form the entirety of the cell's metabolic structure and function in precise terms (Sauer 2006). These modelling approaches serve two purposes - to organise vast amounts of information in a standardised and formal manner; and to obtain the functioning of the metabolic network under multiple environmental and genetic influences. We obtain a tool to carry out in silico experiments under known and novel conditions imposed on the cell.

In this paper, we use a constraint (stoichiometry) based approach (Edwards et al. 2002) to mathematically model the genome scale metabolic network of an endophytic fungus and its host L. perenne (Schardl et al. 2004) and to obtain metabolic fluxes under the hypothesis of evolutionary optimality of metabolic network functioning (Edwards \& Palsson 2000). A novel modelling strategy has been developed to accommodate the effects of a mutant endophyte, a targeted gene replacement of a non-ribosomal peptide synthetase (NRPS-2) from E. festucae, termed $s i d F$, on the symbiotic interactions. NRPS- 2 encodes an extracellular iron-chelating siderophore (Johnson et al. 2007) and the sidF mutant is affected in its iron (Fe) uptake ability. Iron is an essential trace element for all living organisms, but due to insolubility and toxicity, it is not freely available in living tissues. Most fungi overcome this limitation by excreting siderophores with high Fe affinity (Haas 2003). Lack of this mechanism in the sidF KOs resulted in strongly impaired fungal growth under iron limiting conditions in culture. Infection of plants with sidF KOs resulted in a dramatic change in phenotype: fungal growth became de-regulated (branched hyphae, increased fungal biomass), and plant tillers were stunted and sometimes even died - a mutualistic symbiont turned antagonistic (for a detailed description of the phenotypic changes see: Johnson et al. 2007a). We formulate a hypothesis, based on our model and supported by experimental observations that might explain this phenotypic difference.

\section{Modelling Methodology}

Constraint based modelling is suitable for genome scale metabolic networks to characterise metabolic operation numerically, rather than describing just the architectural principles and network organisation. In addition to general characteristics of the network topology like network connectivities, it provides us with throughput rates in various branches of the network operating under steady state. We express the metabolism of an organism in terms of known stoichiometries of the enzyme catalysed reactions. By confining ourselves to steady state operation of the cell, we express the "structure" of the metabolic network in terms of a set of linear algebraic equations, where each of the reaction rates (fluxes) is unknown. Known constraints that arise from thermodynamics (e.g. reversibility or irreversibility of reactions), environment (e.g. constraints on nutrient availability) and genetic modifications (e.g. gene KO) are included as constraints imposed on fluxes. The solution admits a number of metabolic states the organism can function in. We interpret these solutions as the set of all feasible metabolic phenotypes of the organism, which only a gene based approach could not have predicted. A single metabolic state out of the total metabolic capability potentially possible is obtained under the effect of a particular set of genetic and environmental conditions by expressing evolution as an optimisation of certain aspects of the organism/ symbiosis. We thus formulate a Linear Programming problem of mathematics, whose solution is an optimal steady state of metabolic fluxes out of the many feasible ones. The usual hypothesis is that the organism functions in a manner to maximise the flux related to its biomass generation. Hence, there are two mechanisms which determine the steady state the organism functions in - (a) environmental constraints that it operates under, (b) constraints of optimality of metabolic reaction networks. 
The modelling methodology we use has two novel aspects.

1. Handling $s i d F$ KOs of endophyte: Lack of Fe in sidF KOs causes a reduction in the functioning of enzymes that need $\mathrm{Fe}$ as a cofactor. Hence, we target these reactions to impose regulatory constraints in the functioning of the metabolic network. This modelling approach has the advantage of minimally disturbing the structure of the constructed metabolic network for the species under consideration, yet changes in the pathways due to knockouts or varying gene expression and incorporation of regulatory phenomena can be imposed as constraints on the functioning of the network. A list of enzymes that use Fe as co-factor (Table 1) was compiled from the KEGG database KEGG database [KEGG: http://www. genome.jp/kegg/].

2. Handling multiple species: We have extended the methodology to the genome scale metabolic model of two interacting symbiotic species. We distinguish between the common metabolites and reactions that take place in the ryegrass and endophyte cells. "Cross talk" between them is modelled by considering the movement across respective cells as "transport" reactions. The transported molecule loses or gains the identity of the cell it belongs to depending on which of the three "compartments" it finds itself in - fungal cytoplasm, plant cytoplasm, or the intracellular space.

We report here only results obtained for fluxes and concentrations of a small group of metabolites (amino acids), a report on an extended list of metabolites will be presented elsewhere. With regard to 1 , we note that the siderophore itself consists of several amino acids (and derivatives of those), but due to the lack of a confirmed structure we were not able to modify the network at specific parts corresponding to the KO. Such a modification would be a superior method to model direct impacts of reduced production of a certain metabolite (in this case the siderophore) on biosynthetic pathways directly related to this metabolite (e.g. ornithine biosynthesis), but not useful in view of limited information available and also not useful for modelling impacts of the loss of function a specific metabolite might have in the cellular system, e.g. supply of iron as a co-factor necessary for a wide range of enzymatic reactions not related to the biosynthesis of the metabolite (siderophore) itself. We instead use the indirect approach to model the $\mathrm{KO}$ by constraining the fluxes in the reactions where $\mathrm{Fe}$ is involved to low values and report here fluxes obtained for amino acids. With regards to 2, amino acid concentrations were measured by HPLC in extracts obtained from plants infected with fungal wildtype (WT) and mutants, resulting in the inability to differentiate between fungal or plant origin of the observed metabolites.

\section{Results and Discussion}

In absence of genome scale information of metabolism in both N. lolii/ E. festucae and L. perenne, genome scale metabolic networks were constructed separately for endophytes based on $S$. cerevisiae (Famili et al. 2003; KEGG) and for L. perenne based on $A$. thaliana (KEGG). They were combined into a symbiotic network by the use of "cross talk" metabolites transported across their cell boundaries. We assumed all the metabolites involved in transport reactions across cell boundaries as potential "cross talk" molecules, resulting in a total of 116 possible cross talk candidates. From the KEGG database we obtained a list of 11 enzymes whose action could be affected by lack of Fe (Table 1). In our simulations, we constrained these enzymes to act at $50 \%$ of the calculated WT values; it proved to be non-fatal for the organism. A 50\% reduction of enzyme activities was chosen arbitrarily as we have no information on actual constraints reduced levels of iron might have on these enzymes. Organisms usually will not have any problem surviving sub-optimal enzyme activities as they can adjust their metabolism rapidly (Trewavas 2006).

We carried out simulations for WT and sidF KO endophytes in culture, and for the symbiotic network comprising the plant and the two fungal strains. We only have experimental data on metabolite concentrations (rather than fluxes) for fungus and plant pseudo-stem mixtures. Table 2 reports calculated fluxes in amino acid synthesis reactions for the cases of different networks considered (in culture and in planta, WT and KO) and the amino acid concentrations experimentally measured in the associations. The calculations for the fungus in culture show a small decrease in fluxes for the KO. But both calculated fluxes for the integrated ryegrass-endophyte metabolic network and observed mixture concentrations for amino acids are higher for the $\operatorname{sidF} \mathrm{KO}$ as compared to WT.

Based only on the calculations of fungus in culture (column 2 of Table 2), one could postulate that the KO fungus (deficient in iron and suffering oxidative stress) produces less amino acids (reduced fluxes), resulting in less substrate available for e.g. protein biosynthesis and less growth, which is confirmed by the fungal mutant phenotype in culture under iron limiting conditions. As amino acid concentrations were not determined from in culture fungi, we are not able to test this hypothesis on the metabolic level. However, calculations based on plant-fungus symbiotic networks and observations based on amino acid concentrations of the symbiotic association (columns 3 and 4 of Table 2) suggest a different outcome of the sidF $\mathrm{KO}$ in planta. Simulated fluxes for most amino acids are slightly increased and concentrations are higher as well. This could mean that, in the symbiotic association, amino acid biosynthesis is up-regulated under iron stress, but less amino acids are used for downstream biosynthetic pathways (e.g. protein biosynthesis). Taking into consideration that fungal biomass relative to plant biomass increases in the sidF KO- plant association, and that plants infected with these mutants often show a stunted growth phenotype, we propose that the higher levels of amino acids are a result of less use by the plant rather than by the fungus. To test this hypothesis further, an analysis of plant and fungal gene transcript levels related to these metabolic pathways will be performed based on dual Affymetrix gene chips for this association (Cao et al. 2007; Johnson et al. 2007b).

To summarise, we have demonstrated the effectiveness of a systems approach to metabolic modelling since it allows us to obtain fluxes for all the reactions that comprise the organism's (or symbiotic system's) metabolism simultaneously, even when we lack the details of all reaction parameters. The novel aspects of our methodology in modelling metabolism on a genome scale are the manner in which we address (a) symbiotic interactions of metabolic networks of two species and (b) metabolism of genetic KO's by imposing constraints on the pathways these gene products regulate.

\section{Conclusions and Outlook}

The concepts of interdependence between grasses and their endophytes, and indeed symbiosis itself, have been subjected to fresh perspectives recently (Saikkonen et al. 2006; Rasmussen et al. 2007) and it is increasingly being realised that their interactions cannot be explained only in terms of mutual benefits. A fine-tuned balance between the needs and goals of the individual organisms is the pre-requisite for their symbiotic interactions and manipulations of this balance (be it by changes in the environment or by changes of the genetic make-up of 
Table 1 List of selected enzymatic reactions using Fe as co-factor in S. cerevisiae as identified from the KEGG database.

\begin{tabular}{ccll}
\hline $\begin{array}{c}\text { KE } \\
\text { link }\end{array}$ & EC number & Common names (not all shown) & Pathway \\
\hline 1 & 1.1 .1 .100 & closest found: 3-oxoacyl-[acyl-carrier-protein] reductase & \\
7 & 1.13 .11 .10 & 7,8 -dihydroxykynurenate 8,8a-dioxygenase & Tryptophan metabolism \\
25 & 1.13 .11 .5 & homogentisate 1,2-dioxygenase; homogentisicase & Tyrosine metabolism \\
62 & 1.14 .19 .1 & stearoyl-CoA 9-desaturase; delta9-desaturase & fatty acid biosynthesis \\
68 & 1.17 .4 .1 & ribonucleoside-diphosphate reductase & Purine and Pyrimidine metabolism \\
69 & 1.17 .4 .2 & ribonucleoside-triphosphate reductase & Purine and Pyrimidine metabolism \\
76 & 1.3 .5 .1 & succinate dehydrogenase (ubiquinone); succinic deh. & Citric acid cycle \\
77 & 1.3 .99 .1 & succinate dehydrogenase; succinic dehydrogenase & Citric acid and other pathways \\
81 & 1.5 .3 .11 & polyamine oxidase & Polyamine biosynthesis \\
84 & 1.6 .99 .3 & NADH dehydrogenase; cytochrome c reductase & respiration \\
98 & 4.2 .1 .3 & aconitate hydratase; cis-aconitase; aconitase & Citric acid cycle \\
\hline
\end{tabular}

Table 2 Experimental and calculated results for ryegrass infected with WT and sidF KO endophytes, and for the fungal strains in culture. Experimental values are composite average of a mixture of endophyte and plant from pseudostem. Fluxes are normalised with respect to the flux value of 100 for biomass reaction. Note: Trp was not measured experimentally (very low concentrations). Fluxes reported were obtained using the optimisation procedure for biomass.

\begin{tabular}{|c|c|c|c|}
\hline Compound & $\begin{array}{l}\text { Flux in corresponding synthesis } \\
\text { reaction Ratio: Computed KO/ } \\
\text { WT for the fungus in culture }\end{array}$ & $\begin{array}{l}\text { Flux in corresponding synthesis } \\
\text { reaction Ratio: Computed KO/ } \\
\text { WT for the symbiotic network }\end{array}$ & $\begin{array}{l}\text { Amino acid concentration in } \\
\text { planta Ratio: Measured KO/WT }\end{array}$ \\
\hline L-Threonine & 0.9995 & 1.1501 & 1.433481 \\
\hline L-Serine & 0.9968 & 1.1008 & 1.334758 \\
\hline L-Asparagine & 0.9988 & 1.1812 & 1.747075 \\
\hline L-Glutamine & 0.9854 & 1.1805 & 1.647221 \\
\hline L-Proline & 0.9930 & 1.0503 & 1.141393 \\
\hline Glycine & 0.9906 & 1.1804 & 1.661815 \\
\hline L-Alanine & $0 / 0$ & $0 / 0$ & 1.577726 \\
\hline L-Valine & 0.9942 & 1.1796 & 1.655862 \\
\hline L-Cysteine & 1.0210 & 1.3109 & 2.804054 \\
\hline L-Methionine & 1.0100 & 1.0409 & 1.145466 \\
\hline L-Isoleucine & 1.0053 & 1.1701 & 1.411765 \\
\hline L-Leucine & 0.9895 & 1.1906 & 1.754749 \\
\hline L-Tyrosine & 1.0177 & 1.1881 & 1.79613 \\
\hline L-Phenylalanine & 0.9248 & 1.1742 & 1.636669 \\
\hline L-Ornithine & 1.0061 & 1.1003 & 1.726804 \\
\hline L-Lysine & 0.9778 & 1.1870 & 1.700827 \\
\hline L-Histidine & 0.9760 & 1.0200 & 1.296744 \\
\hline L-Arginine & 0.9606 & 1.1817 & 1.837823 \\
\hline L-Tryptophan & 0.6326 & 0.9415 & No experimental values \\
\hline
\end{tabular}

one of the partners) can result in a shift from mutualistic to antagonistic associations.

The ability to produce targeted gene knock-outs of the endophyte, the recent development of dual Affymetrix gene chips for ryegrassendophyte associations and anticipated detailed metabolomics analysis of these associations will allow us to probe further into the intricacies of the ryegrass-endophyte metabolic interactions. These efforts will be greatly facilitated by the availability of the genome sequence of the filamentous fungus Aspergillus niger (EMBL database AM270980-AM270988) and of the soon to be published genome of Epichloë festucae, which will allow us to improve the construction of a metabolic network closer to the endophyte under study.

\section{REFERENCES}

Cao, M.; Johnson, L.; Johnson, R.; Koulman, A.; Lane, G.A.; Rasmussen, S. 2007. Joint analyses of transcriptomic and metabolomic data to probe ryegrass-endophyte symbiosis. pp 195 In: Proceedings of the 6th International Symposium on Fungal Endophytes of Grasses, Grassland Research and Practice Series No. 13. New Zealand Grassland Association.

Edwards, J.S.; Palsson B.O. 2000. The Escherichia coli MG1655 in silico metabolic genotype: Its definition, characteristics, and capabilities. Proceedings of the National Academy of Sciences of the USA 97: 5528-5533.

Edwards, J.S.; Covert, M.; Palsson, B. 2002. Metabolic modelling of microbes: the flux-balance approach. Environmental 
Microbiology 4: 133-140.

Famili, I.; Forster, J.; Nielsen, J.; Palsson, B.O. 2003. Saccharomyces cerevisiae phenotypes can be predicted by using constraint-based analysis of a genome-scale reconstructed metabolic network. Proceedings of the National Academy of Sciences of the USA 100: 13134-13139.

Haas, H. 2003. Molecular genetics of fungal siderophore biosynthesis and uptake: the role of siderophores in iron uptake and storage, Applied Microbiology and Biotechnology 62: 316-330.

Johnson, L.J.; Steringa, A.; Koulman, A.; Christensen, M.; Johnson, R.D.; Voisey, C.R.; Bryan, G.T.; Lamont, I.; Rasmussen, S. 2007a. Biosynthesis of an extracellular siderophore is essential for maintenance of mutualistic endophyte-grass symbioses. pp 177 In: Proceedings of the 6th International Symposium on Fungal Endophytes of Grasses, Grassland Research and Practice Series No. 13. New Zealand Grassland Association.

Johnson, L.J.; Voisey, C.R.; Johnson, R.D.; Khan, A.K.; Park, Z.A.; Ramakrishna, M.; Cao, M.; Simon, D.; Christensen, M.; Bryan, G.T.; Rasmussen, S. 2007b. Dual Affymetrix gene chip analysis of the perennial ryegrass-endophyte symbiosis. pp 509 In: Proceedings of the 6th International Symposium on Fungal Endophytes of Grasses, Grassland Research and Practice Series No. 13. New Zealand Grassland Association.

Mustacchi, R.; Hohmann, S.; Nielsen, J. 2006. Yeast systems biology to unravel the network of life. Yeast 23: 227-238.

Rasmussen, S.; Parsons; A.J.; Bassett, S.; Christensen, M.J.; Hume, D.E.; Johnson, L.J.; Johnson, R.D.; Simpson, W.R.; Stacke, C.; Voisey, C.R.; Xue, H.; Newman, A.J. 2007. High nitrogen supply and carbohydrate content reduce fungal endophyte and alkaloid concentration in Lolium perenne. New Phytologist 173: 787-797.

Saikkonen, K.; Lehtonen, P.; Helander, M.; Koricheva, J.; Faeth, S.H. 2006. Model systems in ecology: dissecting the endophytegrass literature. Trends in Plant Science 11: 428-433.

Sauer, U. 2006. Metabolic networks in motion: ${ }^{13} \mathrm{C}$-based flux analysis. Molecular Systems Biology 62: 1-10.

Schardl, C.; Leuchtmann, L.A.; Spiering, M.J. 2004. Symbioses of grasses with seedborne fungal endophytes. Annual Review of Plant Biology 55: 315- 340.

Trewavas, A. 2006. A Brief History of Systems Biology. Plant Cell 18: 2420-2430. 